\title{
Second Derivative Gaussian Algorithm in Engine Fault Image Yunlin Luo ${ }^{1, \text { a }}$, Guihang Liu', b ${ }^{2}$, Kun Wang ${ }^{3, \text { c }}$ \\ ${ }^{1.2 .3}$ Aeronautical Automation College, Civil Aviation University of China \\ aylluo@cauc.edu.cn, ${ }^{b}$ Igh811@163.com, ${ }^{c k}$ kunwang@cauc.edu.cn
}

Keywords: Second Derivative Gaussian, Wavelet transform, Fault diagnosis

\begin{abstract}
In this paper, we proposed an improved Second Derivative Gaussian (SDG); the endoscopic image of the engine fault was treated by a combination of wavelet and improved SDG, in order to enhance the quality of the image and denosing. The results shows that the propose method can reduce the noise and improve the detail of the image. Provide support for further diagnosis.
\end{abstract}

\section{Introduction}

As an important part of aircrafts, engine's healthy situation directly influences aircraft's security. The engine is working under the harsh environment for a long time, in addition flies the foreign matter hit, will reduce the operating performance, will form the breakage, the safety of the engine caused a great threat. If performs to remove not immediately, bringing the unpredictable serious results. Therefore, the engine must be effectively monitored.

Along with the non-destructive inspection technology's development, endoscopic detection became major engine fault detection. Endoscopic techniques, however there are some birth defects, such as: dark, low-resolution image quality and the inevitable random noise, an image post-processing is necessary. In this paper, Second Derivative Gaussian (SDG) methods are combined with wavelet transform, so as to enhance the quality of endoscopic images to diagnose the engine fault better.

\section{SDG and wavelet transform}

The algorithms of image enhancement, mainly di-vides into the spatial and frequency, these two types have advantages and disadvantages, and the more successful approach is the Gabor filtering proposed by Hong. Gabor wavelet transform is a Gauss Windows and Fourier transform. Daugman in 1980 extended the Gabor wavelet from One-dimensional to two-dimensional, the Gabor filter can simultaneously in the spatial and frequency domain to obtain the best resolution, with continuity and the direction with good selectivity; it can be used Gabor filter in image enhancement. Somsak Choomchuay proposed the SD algorithm, and make success in fingerprint image which has many lines.

Wavelet transform is used for transient and time-varying signals. In the two-dimensional space, a scale function $\varphi(x, y)$ and three directional wavelet $\psi^{H}(x, y), \psi^{V}(x, y), \psi^{D}(x, y)$ is needed.

$$
\begin{aligned}
& \varphi(x, y)=\varphi(x) \varphi(y) \\
& \psi^{H}(x, y)=\psi(x) \varphi(y) \\
& \psi^{V}(x, y)=\varphi(x) \psi(y) \\
& \psi^{D}(x, y)=\psi(x) \psi(y)
\end{aligned}
$$

where, $\psi^{H}(x, y)$ stands for along the column (horizontal) orientation, $\psi^{V}(x, y)$ and $\psi^{D}(x, y)$ measures variations along rows (vertical) and diagonals respectively.

In the two-dimensional discrete wavelet transform, the scale function and wavelet function is defined as: 


$$
\begin{aligned}
& \varphi_{j, m, n}(x, y)=2^{\frac{j}{2}} \varphi\left(2^{j} x-m, 2^{j} y-n\right) \\
& \psi_{j, m, n}(x, y)=2^{\frac{j}{2}} \psi^{i}\left(2^{j} x-m, 2^{j} y-n\right), i=\{H, V, D\}
\end{aligned}
$$

The discrete wavelet transform of function $f(x, y)$ have size of $m \times n$ is defined as:

$$
\begin{aligned}
& W_{\varphi}\left(j_{0}, m, n\right)=\frac{1}{\sqrt{M N}} \sum_{x=0}^{M-1} \sum_{y=0}^{N-1} f(x, y) \varphi_{j_{0}, m, n}(x, y) \\
& W_{\psi}\left(j_{0}, m, n\right)=\frac{1}{\sqrt{M N}} \sum_{x=0}^{M-1} \sum_{y=0}^{N-1} f(x, y) \psi_{j_{0}, m, n}^{i}(x, y), i=\{H, V, D\}
\end{aligned}
$$

In which $f(x, y)$ can be obtained by reversing discrete wavelet:

$$
\begin{aligned}
& f(x, y)= \\
& \frac{1}{\sqrt{M N}} \sum_{m} \sum_{n} W_{\varphi}\left(j_{0}, m, n\right) \varphi_{j_{0}, m, n}(x, y)+\frac{1}{\sqrt{M N}} \sum_{i=H, V, D} \sum_{j=j_{0}}^{\infty} \sum_{m} \sum_{n} W_{\psi}^{i}(j, m, n) \psi_{j, m, n}^{i}(x, y)
\end{aligned}
$$

This paper's approach as shown below:

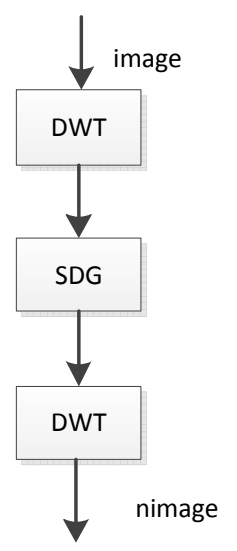

Fig. 1.Approach

Where DWT means directional wavelet transform while nimage stands for the image that we needed.

\section{SDG Algorithms}

In this paper, the image processing as follows:

Normalization. Normalized can reduce the fault edge gray value and can remove the influence of the sensor noise and the gradation distort. Improve the accuracy of edge recognition and reduce the amount of calculation.

Direction Field Estimation. Because of the directional filter is a direction-sensitive filter. Before applying these filters, we must estimate the local orientation of the images.

Before the direction estimation, we use Weiner filter to the image in order to reduce the noise.

We let $\phi(i, j)$ stands for the orientation filed of an engine fault image. $\phi(i, j)$ means the local ridge at pixel $(i, j)$. Generally local ridge is specified for a block rather than for every pixel. Thus, an image is di-vided in to a set of non-overlapping blocks; size of $W \times W$.Each bock holds a single orientation itself. A procedure proposed for orientation estimation is summarized below.

Divide the input image into a series of non-overlapping blocks size of $W \times W$. We used $\mathrm{w}=16$ at this paper. 
At each pixel $(i, j)$, compute the gradient of the $\mathrm{x}$ and $\mathrm{y}$ magnitude, we can gain $\partial_{x}(u, v)$ and $\partial_{y}(u, v)$.

The local orientation of each block which centered at $\operatorname{pixel}(i, j)$ can be estimated by

$$
\phi(i, j)=\frac{1}{2} \tan ^{-1}\left(\frac{V_{y}(i, j)}{V_{x}(i, j)}\right)
$$

The concept of $V_{y}(i, j)$ and $V_{x}(i, j)$ is as follows:

$$
\begin{aligned}
& V_{x}(i, j)=\sum_{u=i-w / 2}^{i+w / 2} \sum_{v=j-w / 2}^{j+w / 2} 2 \partial_{x}(u, v) \partial_{y}(u, v) \\
& V_{y}(i, j)=\sum_{u=i-w / 2}^{i+w / 2} \sum_{v=j-w / 2}^{j+w / 2} 2 \partial^{2}{ }_{x}(u, v) \partial^{2}{ }_{y}(u, v)
\end{aligned}
$$

SDG. A band-pass filter, as long as regulate the frequency and the corresponding direction, can effectively remove the unwanted noise while maintaining good original structure. 2-dimensional Gaussian filters mainly through the smooth image as an image preprocessing denoising step. Second derivative of Gaussian filter is given by the following equation; the formula is a direct extension of the first derivative of Gaussian filter. This can be applied independently to each level.

$$
\begin{aligned}
& S d g(x, y: \phi)=\frac{\left(x_{\phi}^{2}-\sigma_{\phi}^{2}\right)\left(y_{\phi}^{2}-\sigma_{\phi}^{2}\right)}{2 \pi \sigma_{\phi}^{10}} \exp \left(-\frac{x_{\phi}^{2}+y_{\phi}^{2}}{2 \sigma^{2}}\right) \\
& x_{\phi}=x \cos \phi+y \sin \phi \\
& y_{\phi}=-x \sin \phi+y \cos \phi
\end{aligned}
$$
function.

To improve results, we have the style to make simple modifications so it is multiplied by a sine

$$
\operatorname{sdg}(x, y: \phi, f)=\operatorname{sdg}(x, y: \phi) \cdot \cos \left(2 \pi f x_{\phi}\right)
$$

where $\phi$ represents the direction of the second derivative filter field, $f$ is frequency, $\sigma(\sigma=4)$ stands for the standard deviation of the Gaussian. $\mathrm{X}$ and $\mathrm{Y}$ define the $\mathrm{x}$ and $\mathrm{y}$ axis.

\section{Experiment result}

The common injury of aircraft engines are: cracks, nicks, pressure pit, tear, dents, burns, corrosion, Tip curling and so on .In this paper, during the experiment, selected two pictures which are representative from the huge database of engine fault image. They are listed below.

The images above are obtained from endoscopic system. Reflect the engine fault conditions of different parts. Figure 2 is a photo which from turbine while figure 3 represented the condition of combustion chamber. Obviously, the quality of image is dissatisfied, the brightness of the photo is not enough, and fuzzy edges of objects and so on. The clarity of the picture has been greatly enhanced. Edge can be clearly observed. Among them, in the figure 5, the trace in A point is visible, compared to the previous photo, enhance the detection accuracy. Lay a good foundation for further diagnosis. 


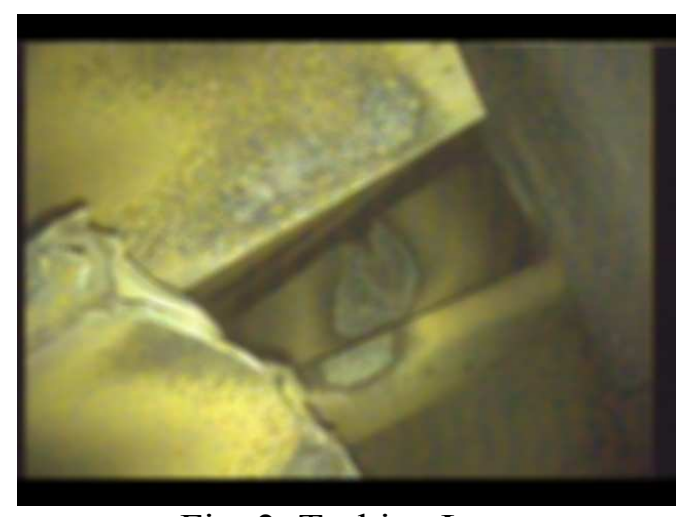

Fig. 2. Turbine Leaves

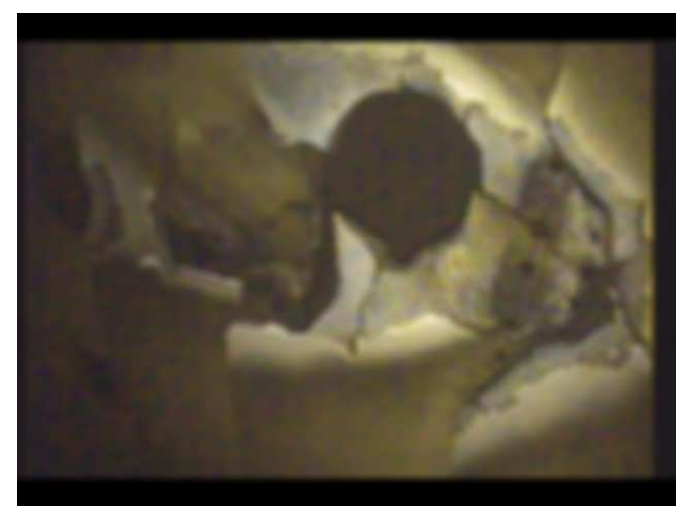

Fig. 4. Combustion chamber

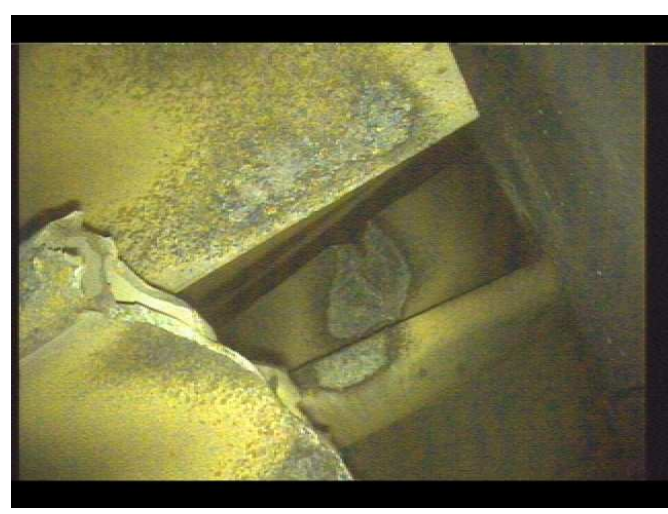

Fig. 3.

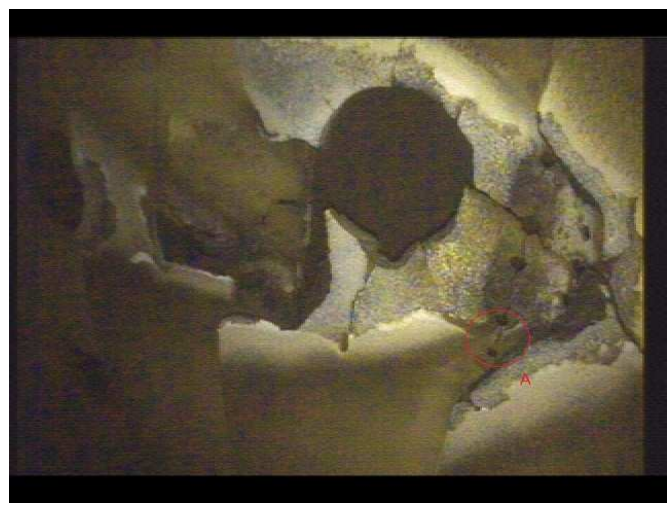

Fig. 5.

\section{Conclusion}

The contribution of this paper is as follows: first, enhance the quality of endoscopic images, help to further diagnostic work. Second, the SDG algorithm is applied to the engine fault image from the endoscopic system. The results demonstrated that the proposed method is feasible and effective. This work is supported by Civil Aviation University of China Science Foundation under Grant 08QD17X, Special Fund for Basic Scientific Research of Central Colleges, ZXH2009C006

\section{References}

[1] Somsak Choomchuay, Keokanlaya Sihalath, an Application of Second Derivative of Gaussian Filters in Fingerprint Image Enhancement, 2010

[2] MENG LIBIN, ZHAO JINCHUANG, FU WENLI, an Improved Fingerprint Enhancement Algorithm Based on Gabor Filter.2006.

[3] Yunlin LUO, Yilong ZHANG, Diagnosis of Aero-engine's Endoscope Faults Based on a New Fuzzy Connectedness, 2009.

[4] Roberto H. Bamberger, a Filter Bank for the Directional Decomposition of Images: Theory and Design, IEEE TRANSACTIONS ON SIGNALPROCESS.1992.

[5] Yuichi Tanaka, Masaaki Ikehara, Multiresolution Image Representation Using Combined 2-D and 1-D Directional Filter Banks,IEEE TRANSACTIONS ON IMAGE PROCESSING, VOL. 18, NO. 2, FEBRUARY 2009.

[6] SU Jin Shan, FENG Yan, A novel DFB of multiresolution and its design

[7] Fingerprint Image Enhancement with Second Derivative Gaussian Filter and Directional WaveletTransform, Keokanlaya Sihalath, Somsak Choomchuay, 2010 Second International Conference on Computer Engineering and Application 